\title{
VISCOSIDADE APARENTE DA POLPA DE MURTA INTEGRAL EM DIFERENTES TEMPERATURAS ${ }^{1}$
}

\author{
REGILANE MARQUES FEITOSA ${ }^{2 *}$, ROSSANA MARIA FEITOSA DE FIGUEIRÊDO ${ }^{3}$, \\ ALEXANDRE JOSÉ DE MELO QUEIROZ ${ }^{3}$, ELISABETE PIANCO DE SOUZA ${ }^{3}$, VIDINA DE MELO SILVA ${ }^{3}$
}

\begin{abstract}
RESUMO - Na industrialização de polpa de frutas frequentemente são utilizados processos térmicos (aquecimento e/ou resfriamento) que podem acarretar modificação na sua viscosidade, o que faz com que o estudo da influência da temperatura sobre o comportamento reológico desse tipo de produto seja de grande importância. Dados da viscosidade aparente de polpas de frutas são utilizados no projeto de equipamentos e na otimização de processos. Diante disso, objetivou-se avaliar a influência da temperatura na viscosidade aparente da polpa da murta. As análises reológicas foram conduzidas em um viscosímetro Brookfield modelo DV-II + Pro. As curvas de viscosidade aparente em função da taxa de deformação foram descritas pelos modelos reológicos de Sisko, Lei da Potência e de Falguera-Ibarz. A polpa da murta integral foi classificada como fluido nãonewtoniano e pseudoplástico. O comportamento deste fluído pode ser bem descrito pelos modelos de Sisko, Lei da Potência e Falguera-Ibarz, com destaque para o modelo de Sisko. O efeito da temperatura sobre a viscosidade aparente foi descrita por uma equação tipo Arrhenius, com a energia de ativação apresentando altos valores à baixas taxas de deformação.
\end{abstract}

Palavras-chave: Eugenia gracillima Kiaersk. Reologia. Arrhenius.

\section{APPARENT VISCOSITY OF MYRTLE PULP INTEGRAL IN DIFFERENT TEMPERATURES}

\begin{abstract}
In fruit pulp industrialization often use thermal processes to (heating and/or cooling) that may cause changes in its viscosity, which causes the study of the influence of temperature on the rheological behavior of this type of product is of great importance. Data of the apparent viscosity of fruit pulp are used in equipment design and process optimization. The research objective was to evaluate the influence of temperature on the apparent viscosity of the Myrtle pulp. The rheological analysis was conducted on a Brookfield viscometer model DV-II + Pro. The apparent viscosity curves as a function of strain rate were described by rheological models Sisko, Power Law, and Falguera-Ibarz. The full Myrtle pulp was classified as non-Newtonian fluid and shear thinning. The behavior of this fluid can be well described by models Sisko, Law of Power and Falguera-Ibarz, especially the Sisko model. The effect of temperature on the apparent viscosity was described by Arrhenius equation with activation energy presenting high values at low shear rate.
\end{abstract}

Keywords: Eugenia gracillima Kiaersk. Rheology. Arrhenius.

\footnotetext{
*Autor para correspondência

${ }^{1}$ Recebido para publicação em 26/03/2014; aceito em 24/08/2015.

Parte da Tese de Doutorado do primeiro autor.

${ }^{2}$ Programa de Pós-Graduação em Engenharia de Processos, UFCG, Campina Grande (PB); regilanemarques@yahoo.com.br.

${ }^{3}$ Unidade Acadêmica de Engenharia Agrícola, UFCG, 58429-970; rossana@deag.ufcg.edu.br, alex@deag.ufcg.edu.br, elisabete_pianco@yahoo.com, vidinamelo@yahoo.com.br.
} 


\section{INTRODUÇ̃̃̃}

O Brasil é um dos três maiores produtores de frutas do mundo, nativas e/ou exóticas, o qual possui um grande potencial nutricional e econômico. As polpas dessas frutas são produzidas e utilizadas na fabricação de novos produtos que estão disponíveis no mercado, mas a produção é geralmente artesanal, em pequena escala e sem procedimentos operacionais adequados (CLERICI; CARVALHO-SILVA, 2011).

Estudos das características reológicas de polpas e demais derivados de frutas em estado fluído ou pastoso, além de ser de suma importância na indústria de alimentos no aperfeiçoamento dos processos e melhorias da qualidade dos produtos resulta na adaptação de sistemas e equipamentos industriais, fornecendo informações fundamentais voltadas para investimentos no segmento agroindustrial (GRANJEIRO et al., 2007).

A mudança nos hábitos alimentares tem levado os consumidores a procura de alimentos nutritivos e exóticos. Dentre as frutas cultivadas e/ou adaptadas no Brasil está a murta (Eugenia gracillima Kiaersk), a qual tem se destacado por suas propriedades de qualidade, com potencial para consumo in natura e processamento agroindustrial e valor relevante de antocianinas (RUFINO et al., 2009; RUFINO et al. 2010). Os alimentos que contem antocianinas têm mostrado benefícios significativos para a saúde e são consideradas como componentes importantes da nutrição humana (FRANK et al., 2012).

A murta, pertencente à família Myrtacea, é uma frutífera não-tradicional, com atrativos e aspectos comerciais praticamente desconhecidos, que permanece subexplorada. A procura dos consumidores por produtos nativos e exóticos está crescendo, em especial para produtos alimentares, criando a necessidade de uma melhor compreensão de seu processamento e propriedades.

É de grande importância viabilizar pesquisas e outros procedimentos voltados para ações que visem a preservação de frutíferas silvestres, como a murta, através da preservação, conservação e valorização dos frutos, tornando-os disponíveis para a sociedade (CRISÓSTOMO et al., 2008).

A viscosidade de polpa de frutas depende de suas características físico-químicas e estrutura e pode ser utilizada como parâmetro de qualidade e correlacionada com a textura e análise sensorial. Na industrialização de polpa de frutas frequentemente são utilizados processos térmicos (aquecimento e/ou resfriamento) que podem acarretar modificação na sua viscosidade, o que faz com que o estudo da influência da temperatura sobre o comportamento reológico desse produto seja de grande importância. Dados de viscosidade em função da temperatura são utilizados no dimensionamento de tubulações, bombas, filtros, agitadores, pasteurizadores, resfriadores, concentradores, congeladores entre outros equipamentos utilizados no processamento de polpas de frutas. Sua determinação além de permitir o correto dimensionamento de equipamentos e otimização de processos contribui com a qualidade das polpas e reduz os custos de produção.

Nesse viés, este trabalho tem por objetivo avaliar a influência da temperatura na viscosidade aparente da polpa da murta.

\section{MATERIAL E MÉTODOS}

A matéria-prima utilizada neste trabalho foi murta (frutos) da espécie Eugenia gracillima Kiaersk, oriunda da Serra dos Paus Dóias, Chapada do Araripe, município de Exu (PE). Os frutos utilizados se encontravam em estádio de maturação maduros, de acordo com a classificação de Araujo (2013), com coloração das cascas da murta com cor $100 \%$ vermelha/violeta. Os frutos foram selecionados manualmente, de forma a eliminar os que apresentavam danos físicos, aspecto de podridão ou em outro estádio de maturação. Posteriormente, submetidos a uma lavagem em água corrente e realizada a sanitização a temperatura ambiente, imergindo-os em recipiente contendo solução de hipoclorito de sódio com concentração de $50 \mathrm{ppm}$ durante 15 minutos. E finalmente enxaguados em água corrente, no tempo de 3 minutos, para retirar o excesso da solução de hipoclorito. Ao ocorrer o escorrimento natural da água de enxágue os frutos foram despolpados em despolpadeira mecânica e a polpa integral embalada em sacos de polietileno de baixa densidade e armazenada em freezer a $-22{ }^{\circ} \mathrm{C}$ até sua utilização nos experimentos.

Para a realização das medidas das viscosidades aparentes da polpa de murta integral, inicialmente a polpa fora descongelada. Em seguida, colocada no porta-amostra do viscosímetro Brookfield (modelo DV-II+Pro), acoplada a um banho termostático para controle da temperatura. Para a realização das leituras utilizou-se o spindle número $28 \mathrm{em}$ diferentes velocidades de rotação $(20,30,40,50,60,70$, $80,90,100,120,140,160,180$ e $200 \mathrm{rpm})$ e temperaturas $\left(15,25\right.$ e $\left.35^{\circ} \mathrm{C}\right)$

Os dados experimentais obtidos foram analisados estatisticamente utilizando-se o programa computacional ASSISTAT versão 7.5 Beta (SILVA; AZEVEDO, 2009), no qual fora aplicado o delineamento inteiramente casualizado em esquema fatorial 14x3, com 14 rotações e 3 temperaturas.

Os modelos reológicos de Sisko (Equação 1), Lei da potência (Equação 2) e Falguera-Ibarz (Equação 3) foram ajustados às curvas da viscosidade aparente em função da taxa de deformação, utilizando-se o programa computacional Statistica para descrever o comportamento reológico da polpa de murta. 


$$
\begin{aligned}
& \eta_{a}=\eta_{\infty}+K_{s} \dot{\gamma}^{\left(n_{s}-1\right)} \\
& \eta_{a}=K \dot{\gamma}^{(n-1)} \\
& \eta_{a}=\eta_{\infty}+\left(\eta_{0}-\eta_{\infty}\right) \cdot \dot{\gamma}^{(-k)}
\end{aligned}
$$

em que:

$$
\begin{aligned}
& \eta_{a} \text { - viscosidade aparente }(\mathrm{Pa} \mathrm{s}) ; \\
& \eta_{\infty} \text { - viscosidade à taxa de cisalhamento }
\end{aligned}
$$
infinita $(\mathrm{Pa} \mathrm{s})$;

$$
K_{s}, K \text { - índice de consistência de Sisko (Pa }
$$

S);

$\dot{\gamma}$ - taxa de deformação $\left(\mathrm{s}^{-1}\right)$;

$n_{s}, \mathrm{n}$ - índice de comportamento do fluído (adimensional);

$\eta_{0}$ - viscosidade aparente estática (Pa s);
$k$ - constante de comportamento do fluído (adimensional).

Foram utilizados como critérios de determinação do melhor ajuste dos modelos aos dados experimentais o coeficiente de determinação $\left(\mathrm{R}^{2}\right)$ e o desvio percentual médio $(\mathrm{P})$, calculado conforme a Equação 4.

$$
\mathrm{P}=\frac{100}{\mathrm{n}} \sum_{\mathrm{i}=1}^{\mathrm{n}} \frac{\left.\mid \mathrm{X}_{\exp }-\mathrm{X}_{\text {teor }}\right) \mid}{\mathrm{X}_{\exp }}
$$

em que:

$\mathrm{P}$ - desvio percentual médio (\%);

$\mathrm{X}_{\text {exp }}$ - valores obtidos experimentalmente;

$\mathrm{X}_{\text {teor }}$ - valores preditos pelo modelo;

$\mathrm{n}$ - número de dados experimentais.

\section{Influência da temperatura}

A influência da temperatura sobre a viscosidade aparente foi avaliada utilizando-se a equação de Arrhenius (Equação 5). Para isso, os valores da viscosidade aparente em função do inverso da temperatura para cada velocidade de rotação $(20,30,40,50$, $60,70,80,90,100,120,140,160,180$ e $200 \mathrm{rpm})$ foram graficados no programa Origin 6.0.

$$
\eta=\eta_{0} \exp \left[-\frac{E_{a}}{R T}\right]
$$

em que:

$\eta$ - viscosidade aparente ( $\mathrm{mPa} \mathrm{s})$;

$\eta_{0}$ - viscosidade aparente inicial ( $\left.\mathrm{mPa} \mathrm{s}\right)$;

$E_{a}$ - energia de ativação $(\mathrm{KJ} / \mathrm{mol})$;

$R$ - constante universal dos gases $(\mathrm{KJ} / \mathrm{mol} \mathrm{K})$;

$\mathrm{T}$ - temperatura $(\mathrm{K})$.

\section{RESULTADOS E DISCUSSÃO}

$\mathrm{Na}$ Tabela 1 estão apresentados os valores médios das viscosidades aparentes da polpa integral da murta em função da velocidade de rotação (20-200 rpm) e temperatura $\left(15,25\right.$ e $\left.35^{\circ} \mathrm{C}\right)$.

Nota-se, para todas as temperaturas $(15,25 \mathrm{e}$ $35^{\circ} \mathrm{C}$ ), tendência de redução da viscosidade aparente com o aumento da velocidade de rotação e com o aumento da temperatura. Foram observadas reduções com o aumento da velocidade de rotação, sendo as referidas reduções estatisticamente significativas entre as velocidades mais baixas. Sengül et al. (2005), ao estudarem o comportamento reológico da polpa de amora nas temperaturas de 30 a $70{ }^{\circ} \mathrm{C}$ e nas velocidades de rotação de 5-100 (rpm), mencionaram que ocorreu diminuição da viscosidade com o aumento da velocidade de rotação e temperatura.

Com relação ao comportamento da viscosidade aparente em relação ao aumento da temperatura, para cada velocidade de rotação foi observado que ocorreram reduções da viscosidade aparente com o aumento da temperatura, sendo estas reduções significativas nas menores velocidades de rotação. Maceiras et al. (2007) afirmaram que existe uma tendência geral para que a viscosidade aparente diminua com o aumento da temperatura, conclusão esta que chegaram ao estudarem o comportamento reológico de diferentes polpas in natura e cozidas (framboesa, morango, pêssego e ameixa) nas temperaturas de 20 $40{ }^{\circ} \mathrm{C}$. Relataram ainda que a viscosidade aparente das polpas diminuíram com o aumento da temperatura. Reduções de viscosidade aparente com o aumento da temperatura também foram reportados por Kechinski et al. (2011), ao estudarem a influência da temperatura $\left(27,40,60,70,80\right.$ e $\left.93{ }^{\circ} \mathrm{C}\right)$ na formulação de polpa de mirtilo adicionada de $2,5 \%$ de goma xantana e $15 \%$ de frutose, por Vandresen et al. (2009), ao aplicarem as temperaturas de 8 a $85^{\circ} \mathrm{C}$ no suco de cenoura, e por Haminiuk et al. (2006), ao verificarem o efeito da temperatura $\left(10\right.$ a $\left.50{ }^{\circ} \mathrm{C}\right)$ na viscosidade da polpa integral de araçá. 
R. M. FEITOSA et al.

Tabela 1. Viscosidades aparentes ( $\mathrm{mPa}$ ) da polpa de murta integral em função da velocidade de rotação e temperatura.

\begin{tabular}{crrr}
\hline $\begin{array}{c}\text { Velocidade de rotação } \\
\text { (rpm) }\end{array}$ & \multicolumn{3}{c}{ Temperatura $\left.\mathbf{~}^{\mathbf{0}} \mathbf{C}\right)$} \\
\cline { 2 - 4 } & $\mathbf{2 5}$ & $\mathbf{2 5}$ & $\mathbf{3 5}$ \\
\hline 20 & $5.475,00 \mathrm{aA}$ & $3.926,66 \mathrm{aB}$ & $2.816,66 \mathrm{aC}$ \\
40 & $3.572,33 \mathrm{bA}$ & $2.821,00 \mathrm{bB}$ & $1.910,00 \mathrm{bC}$ \\
40 & $2.731,66 \mathrm{cA}$ & $2.343,66 \mathrm{cB}$ & $1.596,00 \mathrm{bcC}$ \\
50 & $2.143,33 \mathrm{dA}$ & $1.807,00 \mathrm{~dB}$ & $1.383,33 \mathrm{cdC}$ \\
60 & $1.758,66 \mathrm{eA}$ & $1.447,00 \mathrm{deB}$ & $1.293,66 \mathrm{cdeB}$ \\
70 & $1.520,33 \mathrm{efA}$ & $1.259,66 \mathrm{efB}$ & $1.150,33 \mathrm{defB}$ \\
80 & $1.312,33 \mathrm{fgA}$ & $1.100,00 \mathrm{efgAB}$ & $1.012,43 \mathrm{efgB}$ \\
90 & $1.184,66 \mathrm{fghA}$ & $977,30 \mathrm{fghAB}$ & $921,93 \mathrm{fghB}$ \\
100 & $1.056,66 \mathrm{ghiA}$ & $845,00 \mathrm{ghiA}$ & $841,66 \mathrm{fghiA}$ \\
120 & $934,33 \mathrm{hiA}$ & $713,33 \mathrm{hiA}$ & $720,50 \mathrm{ghiA}$ \\
140 & $875,83 \mathrm{hiA}$ & $609,36 \mathrm{iB}$ & $646,10 \mathrm{hiAB}$ \\
160 & $818,60 \mathrm{iA}$ & $577,60 \mathrm{iA}$ & $587,06 \mathrm{hiA}$ \\
180 & $793,70 \mathrm{iA}$ & $550,93 \mathrm{iAB}$ & $528,46 \mathrm{iB}$ \\
200 & $758,33 \mathrm{iA}$ & $527,33 \mathrm{iAB}$ & $479,00 \mathrm{iB}$ \\
\hline
\end{tabular}

DMS para colunas $=363,762 ;$ DMS para linhas $=251,589 ; \mathrm{MG}=1.436,40 \mathrm{mPa}$; e $\mathrm{CV}=8,98 \%$.

DMS - Desvio mínimo significativo; MG - Média geral; e CV - Coeficiente de variação.

Médias seguidas das mesmas letras minúsculas nas colunas e maiúsculas nas linhas não diferem estatisticamente pelo teste de Tukey a $5 \%$ de probabilidade.

Vários fatores afetam o comportamento reológico das polpas de frutas, destacando-se a temperatura. Para Vidal et al. (2006), comumente a viscosidade das polpas das frutas sofre a influência da temperatura em razão da maioria destas se apresentar na forma de sólidos dispersos em meios líquidos, uma vez que o aumento da temperatura faz com que a viscosidade da fase líquida diminua, aumentando a mobilidade das partículas em suspensão, diminuindo consequentemente a viscosidade da polpa.

$\mathrm{Na}$ Tabela 2 constam os parâmetros reológicos dos modelos de Sisko, Lei da potência e Falguera -Ibarz ajustados aos dados experimentais da viscosidade aparente em função da taxa de deformação da polpa da murta integral nas temperaturas de 15, 25 e $35{ }^{\circ} \mathrm{C}$, da mesma forma como os coeficientes de determinação $\left(\mathrm{R}^{2}\right)$ e os desvios percentuais médios $(\mathrm{P})$. Verifica-se que dentre os modelos testados todos apresentaram bons ajustes, com coeficiente de determinação $\left(R^{2}\right)$ superiores a 0,99 e desvios percentuais médios inferiores a $0,08 \%$. Os resultados indicam bons ajustes dos três modelos e que todos podem ser utilizados na predição do comportamento da viscosidade da polpa da murta integral. Entretanto, os modelos de Falguera-Ibarz e de Sisko apresentaram os melhores ajustes por apresentarem os menores desvios percentuais médios, inferiores a $0,06 \%$. Comportamento semelhante foi verificado por Augusto et al. (2012b) ao aplicarem diversos modelos aos dados obtidos para o suco de tomate processado por homogeneização a alta pressão. O modelo de FalgueraIbarz proporcionou o maior coeficiente de determinação $\left(\mathrm{R}^{2}\right)$, superior a 0,99 .

Segundo Nindo et al. (2007), o modelo de Sisko utiliza três parâmetros e geralmente é aplicado para bombeamento de alimentos fluídos e processos de misturas.

Analisando-se os parâmetros de ajuste do modelo de Sisko, verifica-se que a viscosidade à taxa de cisalhamento infinita $\left(\eta_{\infty}\right)$ apresentou valores negativos nas temperaturas de 25 e $35{ }^{\circ} \mathrm{C}$, indicando que não possuem significado físico neste caso. $\mathrm{Ob}$ servou-se também que ao aumentar a temperatura houve uma redução no índice de consistência $\left(\mathrm{K}_{\mathrm{s}}\right) \mathrm{e}$ o índice de comportamento do fluido $\left(\mathrm{n}_{\mathrm{s}}\right)$ nas temperaturas de 25 e $35{ }^{\circ} \mathrm{C}$ aumentou com o aumento da temperatura, apresentando valores menores que 1 . Para Ahmed et al. (2005), vários estudos têm mostrado que grande parte das polpas de frutas se comporta como um fluído pseudoplástico $(\mathrm{n}<1)$. A característica é fortemente influenciada pelas complexas interações entre os açúcares solúveis, substâncias pécticas e sólidos suspensos.

Valor próximo de $\mathrm{R}^{2}$ foi relatado por Nindo et al. (2007) ao estudarem a influência da temperatura $\left(25,40,50\right.$ e $\left.60^{\circ} \mathrm{C}\right)$ e do conteúdo de sólidos solúveis totais (10 e $\left.25^{\circ} \mathrm{Brix}\right)$ sobre as propriedades reológicas da polpa de mirtilo, observando que a polpa apresentou comportamento pseudoplástico e o comportamento reológico descrito pelo modelo de Sisko $\left(\mathrm{R}^{2}=0,9995\right)$

Quanto ao modelo da Lei da potência, percebe-se que o índice de consistência $(\mathrm{K})$ reduziu ao aumentar a temperatura. Comportamento inverso foi observado no parâmetro $\mathrm{n}$ (índice de comportamento do fluido), que manteve valores inferiores a 1. Resultado semelhante também foi observado por Sengül et al. (2005) para a polpa de amora, empregando as temperaturas de 30 a $70{ }^{\circ} \mathrm{C}$, onde o índice de consistência $(\mathrm{k})$ diminuiu com o aumento da temperatura $\mathrm{e}$ o índice de comportamento do fluido (n) indicou se tratar de fluido pseudoplástico. 
Tabela 2. Parâmetros, coeficientes de determinação $\left(\mathrm{R}^{2}\right)$ e desvios percentuais médios $(\mathrm{P})$ dos modelos reológicos para polpa da murta integral.

\begin{tabular}{|c|c|c|c|c|c|c|}
\hline \multirow{2}{*}{ Modelos } & \multirow{2}{*}{ Temperatura $\left({ }^{\circ} \mathrm{C}\right)$} & \multicolumn{3}{|c|}{ Parâmetros } & \multirow{2}{*}{$\mathbf{R}^{2}$} & \multirow{2}{*}{$\mathbf{P}(\%)$} \\
\hline & & $\eta_{\infty}$ & $\mathbf{k}_{\mathrm{s}}$ & $\mathbf{n}_{\mathrm{s}}$ & & \\
\hline \multirow{3}{*}{ Sisko } & 15 & 339,90 & 38462,3 & $-0,1657$ & 0,9986 & 0,03 \\
\hline & 25 & $-211,165$ & 17039,0 & 0,1857 & 0,9951 & 0,05 \\
\hline & 35 & $-100,89$ & 8734,61 & 0,3322 & 0,9952 & 0,02 \\
\hline \multirow{4}{*}{ Lei da potência } & Temperatura $\left({ }^{\circ} \mathrm{C}\right)$ & $\mathbf{K}$ & & $\mathbf{N}$ & $\mathbf{R}^{2}$ & $\mathbf{P}(\%)$ \\
\hline & 15 & 29314,47 & & 0,0180 & 0,9949 & 0,07 \\
\hline & 25 & 19432,07 & & 0,0856 & 0,9939 & 0,05 \\
\hline & 35 & 9257,04 & & 0,2805 & 0,9949 & 0,03 \\
\hline \multirow{4}{*}{ Falguera-Ibarz } & Temperatura $\left({ }^{\circ} \mathrm{C}\right)$ & $\eta_{\infty}$ & $\eta_{0}$ & $\mathbf{K}$ & $\mathbf{R}^{2}$ & $\mathbf{P}(\%)$ \\
\hline & 15 & 339,90 & 38802,27 & 1,1657 & 0,9986 & 0,03 \\
\hline & 25 & $-211,16$ & 16827,90 & 0,8142 & 0,9951 & 0,05 \\
\hline & 35 & $-100,89$ & 8633,72 & 0,6677 & 0,9952 & 0,00 \\
\hline
\end{tabular}

Para o modelo de Falguera-Ibarz, o aumento da temperatura na polpa de murta integral influenciou os parâmetros estudados $\eta_{\mathrm{o}}$ (viscosidade aparente estática) e $\mathrm{k}$ (constante do comportamento do fluido). A viscosidade aparente $\left(\eta_{\infty}\right)$ apresentou valores negativos, ou seja, sem significado físico para as temperaturas de 25 e $35^{\circ} \mathrm{C}$. A viscosidade aparente estática $\left(\eta_{\mathrm{o}}\right)$ sofreu diminuição com o aumento da temperatu- ra. Augusto et al. (2012a) também verificaram decréscimo para o parâmetro $\eta_{\mathrm{o}}$ ao ajustarem o modelo aos dados do suco de tomate, nas temperaturas de 0 a $80{ }^{\circ} \mathrm{C}$.

A Figura 1 mostra a relação entre a viscosidade aparente e a taxa de deformação a diferentes temperaturas $\left(15,25\right.$ e $\left.35^{\circ} \mathrm{C}\right)$ para polpa integral da murta, com ajustes junto ao modelo de Falguera-Ibarz.

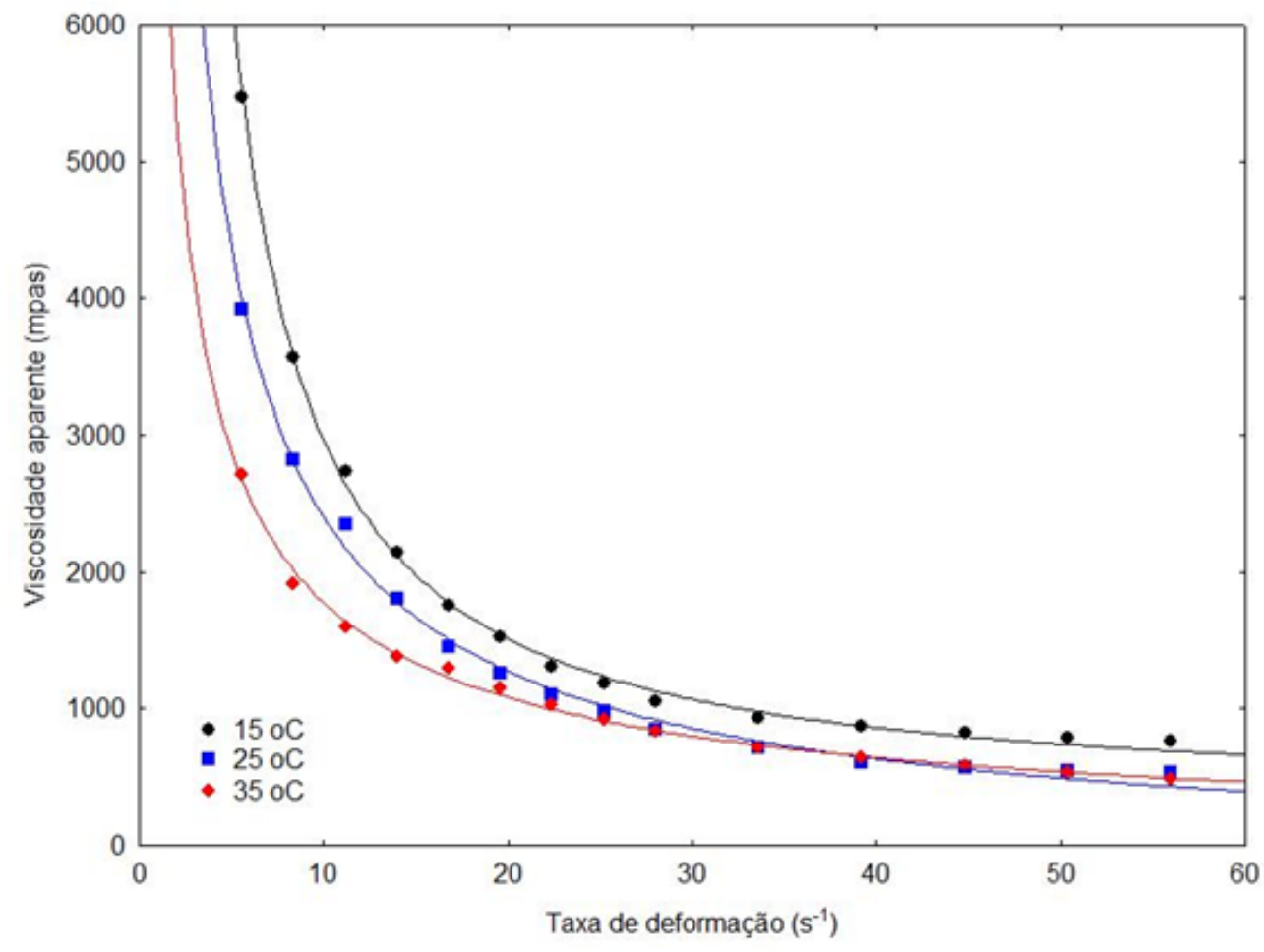

Figura 1. Relação entre a viscosidade aparente e a taxa de deformação da polpa da murta integral ajustada pelo modelo de Falguera-Ibarz. 
Tabela 3. Valores da viscosidade aparente teórica ( $\eta_{0}$ ) e energia de ativação $\left(\mathrm{E}_{\mathrm{a}}\right)$ da polpa de murta integral calculados a partir da equação de Arrhenius.

\begin{tabular}{ccccc}
\hline $\begin{array}{c}\text { Velocidade de } \\
\text { rotação }(\mathbf{r p m})\end{array}$ & $\begin{array}{c}\text { Taxa de deformação } \\
\left(\mathbf{s}^{\mathbf{1}}\right)\end{array}$ & $\eta_{0}(\mathbf{P a ~ s})$ & $\mathbf{E}_{\mathbf{a}}\left(\mathbf{k J} / \mathbf{m o l}^{\mathbf{1}} \mathbf{)}\right.$ & $\mathbf{R}^{\mathbf{2}}$ \\
\hline 20 & 5,6 & 0,1979 & 24,4983 & 0,9997 \\
30 & 8,4 & 0,2458 & 23,0154 & 0,9871 \\
40 & 11,2 & 0,7554 & 19,7138 & 0,9657 \\
50 & 14,0 & 2,6221 & 16,1001 & 0,9893 \\
60 & 16,8 & 15,1665 & 11,3532 & 0,9908 \\
70 & 19,6 & 20,1388 & 10,3196 & 0,9839 \\
80 & 22,4 & 23,4706 & 9,6020 & 0,9827 \\
90 & 25,2 & 23,9007 & 9,2977 & 0,9610 \\
100 & 28,0 & 29,6327 & 8,4759 & 0,8827 \\
120 & 33,6 & 15,5998 & 9,6948 & 0,8592 \\
140 & 39,2 & 7,0515 & 11,3872 & 0,7929 \\
160 & 44,8 & 4,3479 & 12,4053 & 0,8548 \\
180 & 50,4 & 1,3630 & 15,1258 & 0,9167 \\
200 & 56,0 & 0,5899 & 17,0444 & 0,9539 \\
\hline
\end{tabular}

Observa-se que a viscosidade aparente inicial teórica ( $\eta_{0}$ ) aumentou a medida que a energia de ativação diminuiu ao aumentar a taxa de deformação no intervalo de 5,6 a 28,0 s- ${ }^{1}$. Ocorreu efeito contrário a partir da taxa de deformação de $33,6 \mathrm{~s}^{-1}$, dimi-

nuindo a constante $\eta_{0}$ e aumentando a energia de ativação. Efeito semelhante foi observado por Bezerra et al. (2009) ao avaliarem a energia de ativação $\left(\mathrm{E}_{\mathrm{a}}\right)$ da polpa integral de morango, no qual ocorreu redução na energia de ativação com o aumento da taxa de deformação entre 15,8 e $157,8 \mathrm{~s}^{-1}$ e aumento na energia de ativação a partir da taxa de deformação de 221 até $268,2 \mathrm{~s}^{-1}$.

Com o aumento da velocidade de rotação de 100 a $200 \mathrm{rpm}$ na polpa integral da murta ocorreu uma diminuição na viscosidade aparente inicial teóri-

ca $\left(\eta_{0}\right)$ e aumento na energia de ativação $\left(E_{a}\right) . O$ mesmo comportamento foi observado por Miranda et al. (2011) ao aumentarem a velocidade de rotação (50-200 rpm) para a polpa de graviola integral (12 ${ }^{\circ} \mathrm{Brix}$ ), acarretando reduções na viscosidade teórica e tendência de aumento na energia de ativação.

A polpa da murta integral apresenta energia de ativação $\left(E_{a}\right)$ entre 24,4983 e $8,4759 \mathrm{KJ} \mathrm{mol}^{-1}$, em um intervalo da taxa de deformação entre 5,6 e $56 \mathrm{~s}^{-1}$. Valores na faixa dos encontrados para a polpa de murta integral também foram registrados por Dak et al. (2008) para o tomate concentrado $(18,12,18 \mathrm{e}$ $8,4 \%$ de sólidos totais) nas temperaturas de 20,30 , 40,50 e $60{ }^{\circ} \mathrm{C}$, ao encontraram para a energia de ativação valores entre 8,6 e 14,08 kJ/mol. Valor superior ao encontrado neste trabalho para a energia de ativação de $24,4983 \mathrm{~kJ} \mathrm{~mol}^{-1}$ foi obtido por Kaya et al. (2011) para o suco de uva $\left(53,7^{\circ}\right.$ Brix $)$, nas tem- peraturas 5 a $60{ }^{\circ} \mathrm{C}$, com energia de ativação de $26,36 \mathrm{~kJ} \mathrm{~mol}^{-1}$

A energia de ativação de $8,4759\left(\mathrm{~kJ} \mathrm{~mol}^{-1}\right)$ encontrada na faixa de $100 \mathrm{~s}^{-1}$ aproxima-se do valor relatado por Shamsudin et al. (2013) para o suco de abacaxi pasteurizado, que foi de $8,50 \mathrm{~kJ} / \mathrm{mol}$. Para a mesma faixa de taxa de deformação $\left(100 \mathrm{~s}^{-1}\right)$ foram encontrados valores inferiores por Tonon et al. (2009) ao estudarem a energia de ativação da polpa de açaí em diferentes temperaturas $(10,25,40,55$ e $70{ }^{\circ} \mathrm{C}$ ), onde mencionaram valores de 4,18 e $6,21 \mathrm{~kJ} /$ mol.

Comparando a energia de ativação na taxa de deformação de $50,4 \mathrm{~s}^{-1}$, foi reportado por Haminiuk et al. (2006) valor superior em polpa integral de amora-preta, onde verificaram o valor de 18,27 $\mathrm{kJ} / \mathrm{mol}^{-1}$ na taxa de deformação de $50 \mathrm{~s}^{-1}$.

A polpa integral da murta apresenta alta energia de ativação à baixas $\left(5,6\right.$ a $\left.11,2 \mathrm{~s}^{-1}\right)$ taxas de deformação $\mathrm{s}^{-1}$, indicando que a temperatura exerce maior efeito sobre a viscosidade aparente nas baixas taxas de deformação. Para Rigo et al. (2010), os valores da energia de ativação indicam a sensibilidade da viscosidade aparente à alteração da temperatura. De acordo com Ohata e Viotto (2011), altos valores de energia de ativação significam que a viscosidade da polpa é mais sensível a mudança da temperatura.

Os coeficientes de determinação $\left(\mathrm{R}^{2}\right)$ resultaram em valores superiores a 0,78 , os valores próximos a 1,0, indicando que a equação de Arrhenius apresenta bom ajuste aos dados de viscosidade aparente. Silva et al. (2012), ao ajustarem os dados à equação de Arrhenius, obtiveram coeficiente de determinação $\left(\mathrm{R}^{2}\right)$ de 0,85 para a polpa de acerola. Chin et al. (2009) relataram valores de coeficiente de determinação superiores a 0,94 para a equação de Arrhenius aplicada aos dados, 
para a polpa de pomelo em função das concentrações de sólidos solúveis totais 20,30 e $50{ }^{\circ}$ Brix.

Na Figura 2 tem-se a representação gráfica do ajuste da equação de Arrhenius aos dados da viscosidade aparente em função da temperatura da polpa da murta integral.

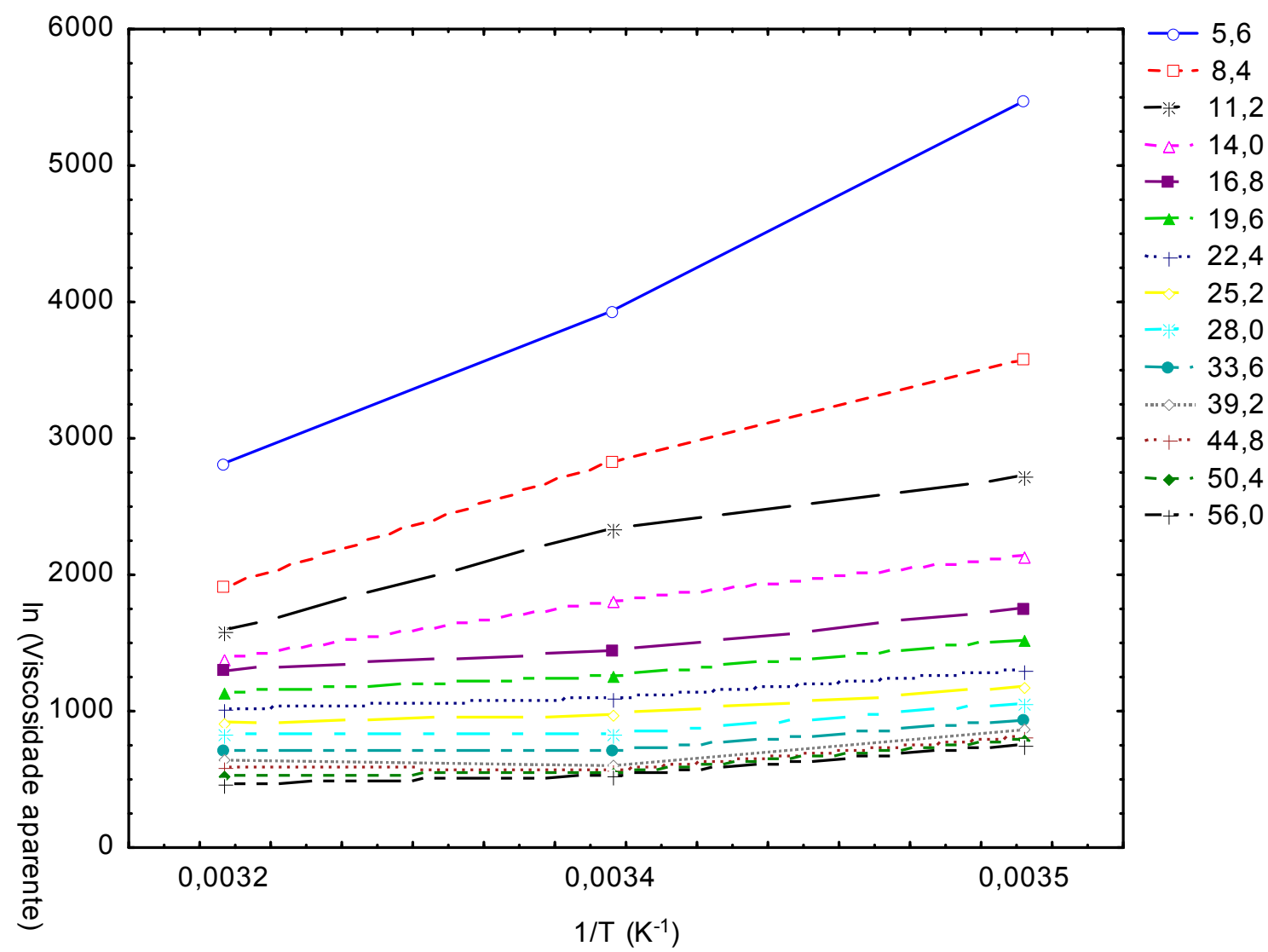

Figura 2. Relação entre a viscosidade aparente e o inverso da temperatura para a polpa da murta integral em diferentes taxas de deformação.

Ao observar a Figura 2 verifica-se que a viscosidade aparente da polpa da murta integral diminui com o aumento da temperatura. Percebe-se também que com o aumento da taxa de deformação a viscosidade aparente também diminui a uma temperatura constante. Oliveira et al. (2009) reportaram o mesmo comportamento na redução da viscosidade aparente da polpa de uva com o aumento da temperatura (20, 25,30 e $35^{\circ} \mathrm{C}$ ) e na taxa de deformação de $264 \mathrm{~s}^{-1} \mathrm{e}$ afirmaram que isso ocorre em razão de que nessa condição a rapidez de movimentação das partículas é mais intensa, enquanto que as forças de interação enfraquecem e as partículas ficam mais livres para escoar.

\section{CONCLUSÃO}

A polpa da murta integral foi classificada como fluido não-newtoniano e pseudoplástico. O comportamento deste fluido pode ser bem descrito pelos modelos de Sisko, Lei da Potência e FalgueraIbarz, com destaque para o modelo de Sisko. O efeito da temperatura sobre a viscosidade aparente foi descrita por uma equação tipo Arrhenius, com a energia de ativação apresentando altos valores à baixas taxas de deformação.

\section{AGRADECIMENTOS}

À Capes pelo apoio financeiro.

\section{REFERÊNCIAS}

AHMED, J.; RAMASWAMY, H. S.; HIREMATH, $\mathrm{N}$. The effect of high pressure treatment on rheological characteristics and colour of mango pulp. Journal of Food Science and Technology, Oxford, v. 40 , n. 8, p. 885-895, 2005.

ARAUJO, D. R. Desenvolvimento, maturidade fisiológica e armazenamento dos frutos da murta. 2013. 158 f. Tese (Doutorado em Engenharia Agrícola: Área de Concentração em Armazenamento e Processamento de Produtos Agrícolas) - Universidade Federal de Campina Grande, Campina Grande, 2013. 
AUGUSTO, P. E. D. et al. Rheological behavior of tomato juice: steady-state shear and time-dependent modeling. Food Bioprocess Technology, Dublin, v. 5, n. 5, p. 1715-1723, 2012a.

AUGUSTO, P. E. D.; IBARZ, A.; CRISTIANINI, M. Effect of high pressure homogenization (HPH) on the rheological properties of tomato juice: Timedependent and steady-state shear. Journal of Food Engineering, London, v. 111, n. 4, p. 570-579, 2012b.

BEZERRA, J. R. M. V. et al. Estudo do efeito da temperatura nas propriedades reológicas da polpa de morango (Fragaria ananassa). Ambiência, Guarapuava, v. 5, n. 1, p. 37-47, 2009.

CLERICI, M. T. P. S.; CARVALHO-SILVA, L. B. Nutritional bioactive compounds and technological aspects of minor fruits grown in Brazil. Food Research International, Canada, v. 44, n. 7, p. 1658$1670,2011$.

CHIN, N. L. et al. Modelling of rheological behaviour of pummelo juice concentrates using mastercurve. Journal of Food Engineering, London, v. 93, n. 2, p. 134-140, 2009

DAK, M.; VERMA, R. C.; JAAFFREY, S. N. A. Rheological properties of tomato concentrate. Journal of Food Engineering, London, v. 4, n. 7, p. 1556 $-3758,2008$.

FALGUERA, V.;IBARZ, A. A new model to describe flow behaviour of concentrated orange juice. Food Biophysics, New Jersey, v. 5, n. 2, p. 114-119, 2010.

GRANGEIRO, A. A. et al. Viscosidades de polpas concentradas de figo-da-índia. Revista Brasileira de Agrociência, Pelotas, v. 13, n. 2, p. 219-224, 2007.

HAMINIUK, C. W. I. et al. Influence of temperature on the rheological behavior of whole aracá pulp (Psidium cattleianum sabine). Food Science and Technology, Oxford, v. 39, n. 4, p. 427-431, 2006.

KAYA, A.; KO, S.; GUNASEKARAN, S. Viscosity and color change during in situ solidification of grape pekmez. Food Bioprocess Technology, Dublin, v. 4, n. 2, p. 241-246, 2011.

KECHINSKI, C. P. et al. Rheological behavior of blueberry (Vaccinium ashei) purees containing xanthan gum and fructose as ingredients. Food Hydrocolloids, Wrexham, v. 25, n. 3, p. 299-306, 2011.

MACEIRAS, R.; ÁLVAREZ, E.; CANCELA, M. A. Rheological properties of fruit purees: effect of cooking. Journal of Food Engineering, London, v. 80, n. 3 , p. $763-769,2007$.

MIRANDA, V. A. M. et al. Viscosidade aparente de polpas de graviola com diferentes concentrações. Revista Brasileira de Produtos Agroindustriais, Campina Grande, v. 13, n. Especial, p. 363-374, 2011.

NINDO, C. I. et al. Rheological properties of blueberry puree for processing applications. Food Science and Technology, Oxford, v. 40, n. 2, p. 292 299, 2007.

OHATA, S. M.; VIOTTO, L. A. Comportamento reológico de constituintes do ovo. Brazilian Journal of Food Technology, Campinas, v. 14, n. 1, p. 10$18,2011$.

OLIVEIRA, R. C.; BARROS, S. T. D.; ROSSI, R. M. Aplicação da metodologia bayesiana para o estudo reológico da polpa de uva. Revista Brasileira de Produtos Agroindustriais, Campina Grande, v. 11, n. 1, p. 73-80, 2009

RIGO, M.; BEZERRA, J. R. M. V.; CÓRDOVA, K. R. V. Estudo do efeito da temperatura nas propriedades reológicas da polpa de butiá (Butia eriospatha). Ambiência, Guarapuava, v. 6, n. 1 p. 25-36, 2010.

RHA, C. Theories and principles of viscosity. In: RHA, C. Theory: determination and control of physical properties of food materials. Dordrecht, The Netherlands: Reidel, 1975.p. 7-249.

RUFINO, M. S. M. et al. Quality for fresh consumption and processing of some non-traditional tropical fruits from Brazil. Fruits, Cambridge, v. 64, n. 6, p. 361-370, 2009.

SENGÜL, M.; ERTUGAY, M. F.; SENGÜL, M. Rheological, physical and chemical characteristics of mulberry pekmez. Food Control, London, v. 16, n. 1, p. 73-76, 2005.

SILVA, L. M. R. et al. Ajuste dos parâmetros reológicos de polpas de acerola, caju e manga em função da temperatura: modelos de Ostwald-deWaelle, Herschel-Bulkley e Casson. Revista Brasileira de Produtos Agroindustriais, Campina Grande, v. 14, n. 1, p. 37-49, 2012.

SHAMSUDIN, R. et al. Rheological properties of ultraviolet-irradiated and thermally pasteurized Yankee pineapple juice. Journal of Food Engineering, London, v. 116, n. 2, p. 548-553, 2013.

TONON, R. V. et al. Steady and dynamic shear rheological properties of açai pulp. Journal of Food Engineering, London, v. 92, n. 4, p. 425-431, 2009. 
VANDRESEN, S. et al. Temperature effect on the rheological behavior of carrot juices. Journal of Food Engineering, London, v. 92, n. 3, p. 269-274, 2009.

VIDAL, J. R. M. B. et al. Propriedades reológicas da polpa de manga (Mangifera indica L. cv. Keitt) centrifugada. Ciência e Agrotecnologia, Lavras, v. 30, n. 5 , p. $955-960,2006$ 